\title{
Automatic Cutting Machine Control System Design For Liquid Steel Funnel
}

\author{
LIU Jian-hua, MA Wen-bin \\ Mechanical Engineering, \\ Changshu institute of technologyChangshu, china
}

Keywords:Motion tracking;System design;Laser cutting

\begin{abstract}
In order to finish the liquid steel funnel work,the laser cutting process have been used to deal with semi-finished product,which has been stretched to given size.The cutting machine control system adopt xin jie PLC and servo moto to finish the cutting system. The whole cutting process involves 3 servo motors linkage, using the given motion curve method solves square tube in the process of laser cutting head and cutting problem for laser surface distance constant, and completed the system design.This paper gives the flow chart of control system design ideas and key subroutine. The system adopts the method of short development cycle, high reliability, has obvious advantages for mass production.
\end{abstract}

\section{Introduction}

The process of liquid steel ladle into the mold from the need in the square after the sprue funnel, as shown in figure 1 . The product production process, using the cutting robot is unable to effectively control the cost, the use of artificial cutting method is difficult to guarantee the quality of. So the factory has designed a set of non-standard cutting equipment. Because the equipment has the advantages of low cost, can effectively save money for orders.

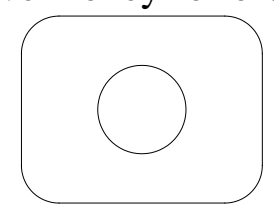

Figure 1 funnel shape sketch

\section{2 .Solution Design}

The upper gate square, the lower part is a circular[1]. After stretching after forming, cutting two positions with a laser. Equipment servo have three, respectively, for driving the work piece rotation, drive work piece moving up and down and drive laser head moves back and forth, mark in the figure were rotary servo, rising servo and feeding servo.

Working principle: First, overview of workers will stretch to complete the cut on the fixture; when the work piece is placed after the completion of the workers, and press the start button, the movable splint clamp to clamp the work piece is clamped in place, if the clamp, the travel switch will move, otherwise the clamping error; secondly control system gets the signal clamping completed after the start, mobile feed servo, makes the laser head from the cutting position 1 is $5 \mathrm{~mm}$, when the feed servo moving in place, start the laser cutting start, at the same time at a given speed start rotation servo rotation, rotation of $360^{\circ}$, cut finish gate circle lower, at this time the laser head servo after the move to stop working, the origin position; once again, rising servo start, drives the rotating mechanism up to a square opening cutting position. This rise in servo through power locking. Feed servo start again, to be laser head distance work piece surface $5 \mathrm{~mm}$, laser cutting start, start at the same time rotary servo; because the work piece to be cut into the shape of a square, so that the work piece rotates feed servo, to ensure that the laser head and the work piece surface distance. When the rotary servo rotation to 360 degrees, the laser head to stop, back to the origin of the feed servo. Finally, the rise began to reverse the origin position servo until the rise, then the 
dynamic splint loosening, workers remove the work piece[2] finished from the device.

\section{The design of key subsystem}

\subsection{System overview}

The design of the system has three degrees of freedom, respectively is: the rise and fall of freedom, freedom degree of rotation and laser head feeding freedom; three degrees of freedom by three servo motor control. Three degree of freedom system there are three origin position, the origin position 1 is starting origin servo system is also rising, stop the origin; the origin position 2 is the origin of the rotation of rotary servo, because every job is the entire week rotation, so the origin is moving relative to the origin, in case of emergency needs calibration, can enter the single step operation state, correction; origin position 3 for feeding the origin, the starting point and end point of the work is the position of the laser head; above the origin position need to re correction can single step through manual operation correction.

System wiring diagram as shown in figure 2. Among them, PLC select Xin jie XCM motion control PLC, to support the common motion control function; the project selection model: XCM-32T4, which can support up to 4 servo motors, for equipment expansion left a certain space[3]; servo motor and servo driver also choose Xin jie DS2 series servo[4]; the touch screen to choose the TH series industrial touch screen; a travel switch and a proximity switch select OMRON series. The key point of the whole system is: square gate cutting position 2 laser head in the rotation process according to the need of mobile trajectory, meet the laser head and liquid steel gate location does not exceed $5 \mathrm{~mm}$. The subroutine design process needs to complete the given trajectory control.

Figure $2 \mathrm{KV}$ is pneumatic clamping electromagnetic valve,KA as the intermediate relay to control the laser head laser emission.

\subsection{The design of key subsystem}

The key to the whole control system is to control the cutting distance square gate, ensure the work piece to be cut when the square in the process of rotation, the laser head cutting surface distance of not more than $5 \mathrm{~mm}$.

A way to solve this problem is through the front of a displacement sensor, closed-loop system is formed by the sensor, to complete the follow-up process.

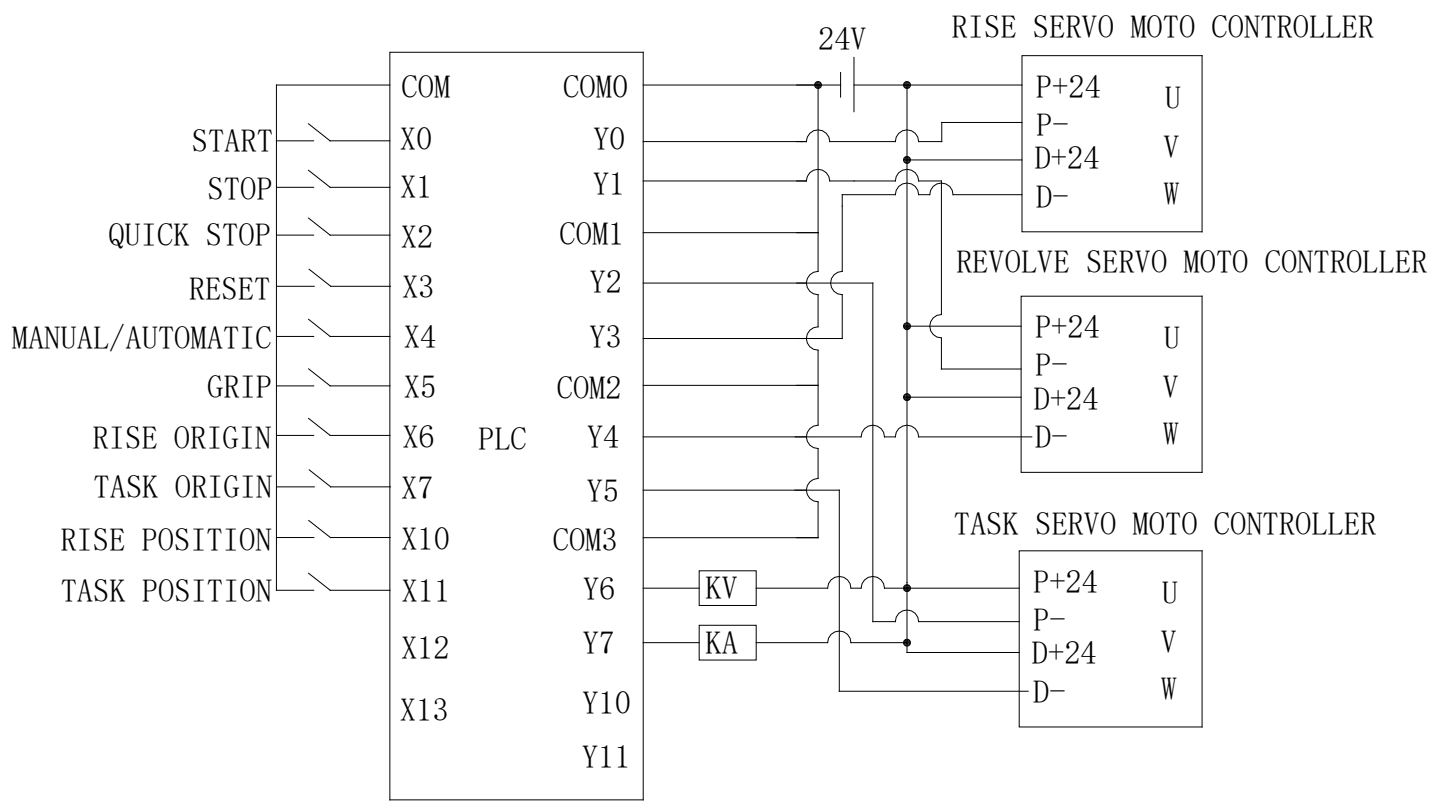

Figure 2 system wiring diagram

But with double PID method, in the actual use of the process found that not only the reliability is difficult to guarantee, the two parameter PID values are given for stabilization of the difficult in a 
short period of time.

Consider a single sex to the product, using the open loop system, which makes the laser head according to the contour curve move given, not only can shorten system development cycle, and high reliability.

Using the method given contour curve relates to the linkage rotary servo servo. Therefore, in the program design process should be the first analysis of the characteristics of the work piece contour curve.Circular chamfer radius is $5 \mathrm{~mm}$.

As you can see from Figure 1, gate shape are arranged symmetrically, the gate shape can be divided into two parts totally the same. Only needs to solve the cutting angle can be a part of another corner solutions.

Through the actual measurement, a prominent position in the fillet of the relative height difference of maximum up to $3 \mathrm{~mm}$. Thus, assume complete follow-up control a prominent rounded corners, the remaining control thought the same.

The design idea in Figure 3: each corner of the program for the calculation of servo movement distance and servo movement frequencies, sends out pulse process is separate process, starting conditions for auxiliary relay $\mathrm{M}$, therefore, in the above key subroutines in geometric computing shape for the way of calculation, due to the length of the relationship, most programs do not describe.

\section{4 .Sequence control}

The control objectives and requirements of the control system in the form of a flowchart represents the control sequence, from different processes can be seen in the control of the whole system and the requirement of control process.

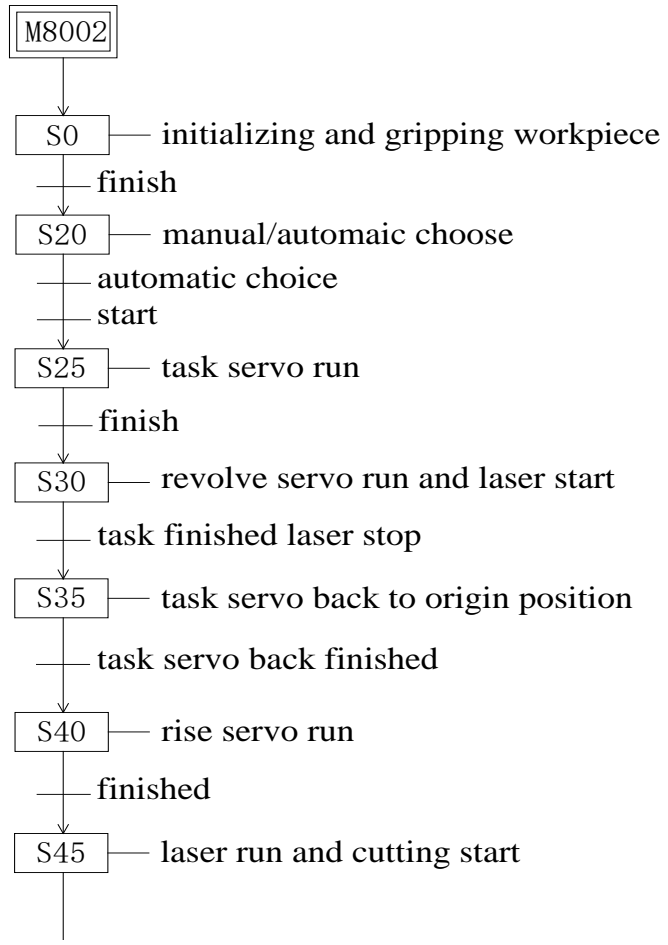

Figure. 3 part program flow

The control process of the system is shown in Figure 4, which mainly describes the automatic control process, each process manual control process for parallel relationship.

\section{Summary}

This paper introduces a cutting equipment automatic system design process for the liquid steel gate.Due to the adoption of the open loop control system based on the contour, the high reliability, short development cycle, lower cost. Obvious advantages for large quantities of single part 
production.

\section{References}

[1] Liu Jie,Yu Hui-qun.Pressure system design steel making furnace based on the PLC controller. MANUFACTURING AUTOMATION. Vol.35(4), 2013.4: 140-143

[2] Wang Hai-xiang,Gao Feng.The control system design of cutting plate machine based on the PLC and servo system.MANUFACTURING AUTOMATION.Vol.34(12),2012(12):115-117;

[3] Zaheer-uddin M,Zheng G R.A dynamic model of a mulit-zone VAV system for control analsisi.ASHRAE Trans 1994,100(1):219-229

[4] Akhyar,S.,Omatu S. Self-tuning PID control by neural-networks.IEEE Transactions on Power Systems, Vol 3,25-29 Oct 1993:2749-2752

[5] Zhang Jie,Zou Ji-gang,Li Wen-xiu.PID neural Network for Decoupling Control of Multi-input and Multi-output system.Journal of Harbin engineering University.Vol.21(5)2000(10):6-9

[6] Li Guo-ping,Zhang Qing-wei. Research on Fault Diagnosis for Hydraulic Excavator Based on BP neural Network. Machine Tool \& hydraulics. Vo.l39(23), 2011(12):160-164 